\title{
A NOTE ON THE PROBLEM OF LAGRANGE IN THE CALCULUS OF VARIATIONS.
}

\author{
BY PROFESSOR GILBERT AMES BLISS.
}

(Read before the American Mathematical Society, December 31, 1915.)

There are two theorems concerning the solutions of a system of linear differential equations, due to von Escherich and A. Mayer,* which play an important rôle in the proofs of the sufficient conditions for a problem of the calculus of variations in the form proposed by Lagrange. Bolza remarks $\dagger$ that the theory of the second variation has so far been essential to the establishment of these two theorems, as well as to the proof of the necessity of Jacobi's condition in the exceptional cases not covered by the geometrical theory of Kneser. In a paper which will appear in the near future, the writer has shown that for problems in parametric form in any number of dimensions an inclusive proof of the Jacobi condition may be made very simply by an application of Euler's equations and the usual corner point condition to the second variation. A similar result has been attained for the problem of Lagrange by D. M. Smith. It is desirable therefore to have deductions of the two theorems mentioned above which also shall be independent of the complicated transformations of the second variation. The proofs given below have this advantage.

The form $\ddagger$

\section{§1. The Differential Equations.}

$$
\begin{array}{r}
2 \Omega\left(\eta, \eta^{\prime}, \mu\right)=\sum_{i, k}\left(P_{i k} \eta_{i} \eta_{k}+2 Q_{i k} \eta_{i} \eta_{k}{ }^{\prime}+R_{i k} \eta_{i}{ }^{\prime} \eta_{k}{ }^{\prime}\right)+ \\
\sum_{\beta, i} \mu_{\beta}\left(S_{\beta i} \eta_{i}+T_{\beta i} \eta_{i}{ }^{\prime}\right),
\end{array}
$$

where $i, k, \beta$ have the ranges

$$
i, k=1,2, \cdots, n ; \quad \beta=1,2, \cdots, m ; \quad m<n,
$$

is quadratic and homogeneous in the variables $\eta, \eta^{\prime}, \mu$, with coefficients and variables which are supposed to be functions of $x$ of class $C^{\prime}$ on an interval $x_{1} \leqq x \leqq x_{2}$. The prime denotes

\footnotetext{
* See Bolza, Vorlesungen über Variationsrechnung, p. 633.

- Loc. cit., p. 634.

$¥$ Bolza, loc. cit., p. 621 .
} 
differentiation with respect to $x$. In the sequel it will be convenient to represent multipartite numbers and matrices by single symbols, with the usual agreements as to the meanings of their products.* The expression for $\Omega$ would then be

$$
2 \Omega\left(\eta, \eta^{\prime}, \mu\right)=P \eta \cdot \eta+2 Q \eta \cdot \eta^{\prime}+R \eta^{\prime} \cdot \eta^{\prime}+2 S \eta \cdot \mu+2 T \eta^{\prime} \cdot \mu \text {. }
$$

The matrices $P$ and $R$ are symmetric.

The system of $m+n$ linear differential equations to be considered can now be represented in the form

$$
\Phi(\eta)=\Omega_{\mu}=0, \quad \Psi(\eta, \mu)=\Omega_{\eta}-\frac{d}{d x} \Omega_{\eta^{\prime}}=0,
$$

where the symbol $\Omega_{\mu}$, for example, represents the multipartite number $\left(\partial \Omega / \partial \mu_{1}, \cdots, \partial \Omega / \partial \mu_{m}\right)$. A multipartite number is by definition equal to zero only when every element is zero. There are therefore $m$ of the former equations and $n$ of the latter.

The quadratic form $\Omega$ satisfies the well-known relations

$$
\begin{aligned}
& \eta \cdot \Omega_{\eta}+\eta^{\prime} \cdot \Omega_{\eta^{\prime}}+\mu \cdot \Omega_{\mu}=2 \Omega, \\
& \eta \cdot \Omega_{u}+\eta^{\prime} \cdot \Omega_{u^{\prime}}+\mu \cdot \Omega_{\sigma}=u \cdot \Omega_{\eta}+u^{\prime} \cdot \Omega_{\eta^{\prime}}+\sigma \cdot \Omega_{\mu},
\end{aligned}
$$

where $(u, \sigma)$ is a second set of arguments of the type $(\eta, \mu)$, and $\Omega_{u}$, for example, is the row of derivatives of the function $\Omega\left(u, u^{\prime}, \sigma\right)$ with respect to the elements of $u$. The product, indicated by a dot, of two multipartite numbers such as $\eta$ and $\Omega_{u}$, is the sum of the products of their respective elements. As a result of the second of the relations (2) one finds the formula

$u \cdot \Psi(\eta, u)-\eta \cdot \Psi(u, \sigma)+\sigma \cdot \Phi(\eta)-\mu \cdot \Phi(u)=\frac{d}{d x}\left(\eta \cdot \Omega_{u^{\prime}}-u \cdot \Omega_{\eta^{\prime}}\right)$.

* The notations and properties used here are very simple. The symbols $\eta, \eta^{\prime}, \mu$, for example, denote the multipartite numbers

$$
\eta=\left(\eta_{1}, \eta_{2}, \cdots, \eta_{n}\right), \eta^{\prime}=\left(\eta_{1}{ }^{\prime}, \eta_{2}{ }^{\prime}, \cdots, \eta_{n}{ }^{\prime}\right), \mu=\left(\mu_{1}, \mu_{2}, \cdots, \mu_{m}\right),
$$

and $Q$ is the matrix of elements $Q_{i k}(i, k=1,2, \cdots, n)$. Then $Q_{\eta}$ is by definition a multipartite number

$$
Q_{\eta}=\left(\Sigma Q_{1 k \eta}, \Sigma Q_{2 k} \eta_{k}, \cdots, \Sigma Q_{n k} \eta_{k}\right),
$$

and $Q \eta \cdot \eta^{\prime}$ is the bilinear expression found by multiplying each element of $Q \eta$ by the corresponding element of $\eta^{\prime}$ and adding, according to the usual definition of scalar multiplication. If further details seem to be necessary for the developments of the present paper, see Bliss, "The solutions of differential equations of the first order as functions of their initial values," Annals of Mathematics, 2d series, vol. 6 (1905), p. 58. 
Every pair of solutions $(\eta, \mu),(u, \sigma)$ of the equations (1) clearly makes the last expression in parenthesis have a constant value. When this constant is zero the two solutions are said to be conjugate.

A conjugate system of solutions is a system of $n$ linearly independent solutions every two of which are conjugate. The functions $u, \sigma$ belonging to such a system are the elements of two matrices $U, \Sigma$, the former of which has $n$ columns and rows, while the latter has $n$ columns and $m$ rows. A column of $U$ with the corresponding column of $\Sigma$ forms a solution of type $(\eta, \mu)$ of the equations (1). The solutions $(u, \sigma),(v, \tau)$ defined by the equations

$$
u=U c, \quad \sigma=\Sigma c ; \quad v=U d, \quad \tau=\Sigma d,
$$

where $c$ and $d$ are constant multipartite numbers of $n$ elements, satisfy the relation

$$
u \cdot \Omega_{v^{\prime}}-v \cdot \Omega_{u^{\prime}}=0,
$$

since a similar relation holds for every pair of the solutions of the conjugate system $U, \Sigma$. It is important to note that the equation (3) is an identity in the elements of $c$ and $d$, and would remain true even if these elements were functions of $x$.

\section{$\S 2$. The Proofs of the Theorems in Question.}

Let $(\eta, \mu)$ be an arbitrarily selected set of functions of the type described in the preceding section. If $U, \Sigma$ is a conjugate system of solutions with determinant $|U|$ different from zero on the interval $x_{1} \leqq x \leqq x_{2}$, the linear equations

$$
\eta=U c
$$

determine uniquely the elements of $c$ as functions of $x$; in other words, there passes a unique solution

$$
u=U c, \quad \sigma=\Sigma c
$$

through each point of the curve defined by the functions $\eta$ in the $(n+1)$-dimensional $x \eta$-space.

Lemma. If the system $(\eta, \mu)$ is a solution of the equations (1), then in every interval $x_{1} \leqq x \leqq x_{2}$ for which the determinant of the matrix $U$ is different from zero

$$
\frac{d}{d x} \eta \cdot\left(\Omega_{\eta^{\prime}}-\Omega_{u^{\prime}}\right)=R\left(\eta^{\prime}-u^{\prime}\right) \cdot\left(\eta^{\prime}-u^{\prime}\right) .
$$


For let $(v, \tau)$ be the solution

$$
v=U c^{\prime}, \tau=\Sigma c^{\prime}
$$

defined at each point of the $x \eta$-curve by the derivatives of the functions $c$ determined by equations (4). With the belp of the first of the relations

$$
u^{\prime}=U^{\prime} c, v^{\prime}=U^{\prime} c^{\prime}
$$

it follows from (4) by differentiation that

$$
\eta^{\prime}=U^{\prime} c+U c^{\prime}=u^{\prime}+v .
$$

Now

$\frac{d}{d x} \eta \cdot\left(\Omega_{\eta^{\prime}}-\Omega_{u^{\prime}}\right)=\eta^{\prime} \cdot\left(\Omega_{\eta^{\prime}}-\Omega_{u^{\prime}}\right)+\eta \cdot\left(\frac{d}{d x} \Omega_{\eta^{\prime}}-\frac{\partial}{\partial x} \Omega_{u^{\prime}}-\Omega_{u^{\prime} c} \cdot c^{\prime}\right)$,

where the symbol of partial differentiation indicates that during that operation the elements of $c$ are regarded as constants. The expression $\Omega_{u^{\prime}}$ is linear in the elements of $c$, and the expression $\Omega_{u^{\prime} c} \cdot c^{\prime}$ is therefore precisely $\Omega_{u^{\prime}}$ with $c$ replaced by $c^{\prime}$, that is, $\Omega_{v}$. The last equation may therefore be written, with the help of equations (1) and the relations $\eta=u, \eta^{\prime}=u^{\prime}+v$, in the form

$$
\begin{aligned}
\frac{d}{d x} \eta \cdot\left(\Omega_{\eta^{\prime}}-\Omega_{u^{\prime}}\right)= & \eta^{\prime} \cdot\left(\Omega_{\eta^{\prime}}-\Omega_{u^{\prime}}\right)+\eta \cdot\left(\Omega_{\eta}-\Omega_{u}-\Omega_{v^{\prime}}\right) \\
= & \eta \cdot \Omega_{\eta}+\eta^{\prime} \cdot \Omega_{\eta^{\prime}}-u \cdot \Omega_{u}-u^{\prime} \cdot \Omega_{u^{\prime}} \\
& \quad-2\left(\eta^{\prime}-u^{\prime}\right) \cdot \Omega_{u^{\prime}}-u \cdot \Omega_{v^{\prime}}+v \cdot \Omega_{u^{\prime}} .
\end{aligned}
$$

But equation (3) holds even when the values $c$ and $d=c^{\prime}$ are functions of $x$, and since $\Omega_{\mu}=\Omega_{\sigma}=0$ the formulas (2) give $\frac{d}{d x} \eta \cdot\left(\Omega_{\eta^{\prime}}-\Omega_{u^{\prime}}\right)=2 \Omega\left(\eta, \eta^{\prime}, \mu\right)-2 \Omega\left(u, u^{\prime}, \sigma\right)-2\left(\eta^{\prime}-u^{\prime}\right) \cdot \Omega_{u^{\prime}}$

The expression on the right is readily identified with the second member of (5) by an application of Taylor's formula. For since $\eta=u$

$$
\begin{aligned}
& \Omega\left(\eta, \eta^{\prime}, \mu\right)=\Omega\left(u, u^{\prime}, \sigma\right)+\Omega_{u^{\prime}} \cdot\left(\eta^{\prime}-u^{\prime}\right)+\Omega_{\sigma} \cdot(\mu-\sigma) \\
& +\frac{1}{2} \Omega_{u^{\prime} w^{\prime}}\left(\eta^{\prime}-u^{\prime}\right) \cdot\left(\eta^{\prime}-u^{\prime}\right)+\Omega_{\sigma u^{\prime}}\left(\eta^{\prime}-u^{\prime}\right) \cdot(\mu-\sigma) .
\end{aligned}
$$

The third term on the right evidently vanishes, and the last also because

$$
0=\Omega_{\mu}-\Omega_{\sigma}=\Omega_{\sigma u^{\prime}}\left(\eta^{\prime}-u^{\prime}\right) .
$$


The matrix $\Omega_{u^{\prime} u^{\prime}}$ is precisely the matrix $R$.

Let $V, W$ be two matrices of order $n$ whose columns belong to solutions of equations (1), as described in the preceding paragraphs, without being necessarily conjugate to each other. Let $\Delta(x, \xi)$ represent the determinant of order $2 n$

$$
\Delta(x, \xi)=\left|\begin{array}{ll}
V(x) & W(x) \\
V(\xi) & W(\xi)
\end{array}\right| .
$$

The first of the two theorems mentioned is then as follows:

If at every point of an interval $x_{1} \leqq x \leqq x_{2}$ the quadratic form with matrix $R$ is positive definite, and if furthermore there exists a conjugate system $U$ with determinant $|U| \neq 0$ on the interval, then on the same interval the determinant $\Delta(x, \xi)$ vanishes only at $x=\xi$ or else is identically zero.

Under the hypothesis of the theorem a solution $(\eta, \mu)$ of equations (1) having elements $\eta$ vanishing simultaneously at two points $\xi$ and $\xi^{\prime}$ of the interval in question must be identically zero. For the derivative of the function $\eta \cdot\left(\Omega_{\eta^{\prime}}-\Omega_{u^{\prime}}\right)$ expressed by formula (5) is clearly positive or zero between $\xi$ and $\xi^{\prime}$ since the quadratic form with matrix $R$ is positive definite. If the elements of $\eta$ vanish at $\xi$ and $\xi^{\prime}$, so does the function $\eta \cdot\left(\Omega_{\eta^{\prime}}-\Omega_{u^{\prime}}\right)$, and the derivative of this function must be identically zero between $\xi$ and $\xi^{\prime}$. In that case the elements of $\eta^{\prime}-u^{\prime}=v$ are identically zero, and equations (7) and (6) show that the functions $c$ are constant. The elements of $\eta$ can not then vanish at all unless the elements of $c$ are all zero, since the determinant of the matrix $U$ in equations (4) is different from zero.

If $\Delta\left(\xi^{\prime}, \xi\right)=0$ for a value $\xi^{\prime} \neq \xi$, it is always possible to find a set of functions

$$
\eta(x)=V(x) \cdot c+W(x) \cdot d
$$

linear in the $2 n$ constants $c$ and $d$ and vanishing at $\xi$ and $\xi^{\prime}$. These functions $\eta$ belong to solutions of the linear equations (1), and by the reasoning of the preceding paragraph must be identically zero. But in this case it follows readily that $\Delta(x, \xi) \equiv 0$ since $c, d$ are not both zero.

It can be shown that for every point $\xi$ there exists a matrix $U$ of conjugate solutions with determinant different from zero at $\xi$.* The last theorem then shows that the zero $x=\xi$

* Von Escherich, Wiener Berichte, vol. 108 (1899), p. 1339; Bolza, loc. cit., p. 627. 
of the determinant $\Delta(x, \xi)$ is an isolated one. This is the second theorem of Bolza mentioned in the introduction above.

The formula (5) is identical with a formula of von Escherich* when the values of $c$ from equations (4) are substituted and $\eta$ replaced by $z$. The proof here is, however, of an entirely different character and by far more simple than his. Bolza uses the formula of von Escherich for the purpose of transforming the second variation, and with the help of this transformation deduces the theorem last given. This process seems very much less direct than the argument given above.

University of Chicago.

\section{CONCERNING A NON-METRICAL PSEUDO- ARCHIMEDEAN AXIOM.}

BY DR. ROBERT L. MOORE.

(Read before the American Mathematical Society, April 26, 1913.)

\section{$\S 1$. Introduction.}

LET $H_{1}$ denote Hilbert's plane Axioms of Groups I and II $\dagger$ or Veblen's Axioms I-VIII. $\ddagger$ Let $H_{2}$ denote $H_{1}$ together with Desargues' theorem§ (considered as an axiom) and Hilbert's III (axiom of parallels). It is well known that if a twodimensional space $S$ satisfies $H_{2}$ together with Hilbert's congruence axioms of Group IV and the archimedean axiom that of any two non-congruent segments some multiple of the smaller is larger than the greater, then $S$ is either an ordinary euclidean space of two dimensions or an everywhere dense subset of such a space.

Consider the following non-metrical pseudo-archimedean axiom:

Axıом A. If (1) the points of a line $l$ (Fig. 1) are divided into two sets $S_{1}$ and $S_{2}$ such that no point of either of these sets is between two points of the other one and such that no point $P$ is

* Loc. cit., p. 1283, formula (9); Bolza, loc. cit., p. 630, formula (68).

$\dagger$ D. Hilbert, Foundations of Geometry, translated by E. J. Townsend, Open Court Publishing Co., Chicago, 1902.

† O. Veblen, "A system of axioms for geometry," Transactions Amer. Math. Society, vol. 5 (1904), pp. 343-384.

\& Cf. Hilbert, loc. cit., p. 71 . 